\title{
Differential relationship between physical activity and progression to diabetes by glucose tolerance status: the Inter99 Study
}

\author{
S. Engberg • C. Glümer • D. R. Witte • T. Jørgensen • \\ K. Borch-Johnsen
}

Received: 3 July 2009 / Accepted: 6 October 2009/Published online: 7 November 2009

(C) Springer-Verlag 2009

\begin{abstract}
Aims/hypothesis The aim of the study was to analyse how strongly commuting and leisure-time physical activity affect progression to diabetes and to study whether this relationship is different in individuals with isolated impaired fasting glucose (i-IFG) and isolated impaired glucose tolerance (i-IGT).

Methods We studied the incidence of diabetes in 4,031 individuals without diabetes at baseline who participated in the baseline and 5 year follow-up examinations of a population-based primary prevention study, the Inter99 Study. Glucose tolerance status at baseline and at followup were based on OGTTs. Commuting and leisure-time physical activity at baseline were assessed by questionnaire. We present rate ratios from Poisson regression analyses adjusted for relevant confounders.

Results The progression rate to diabetes was lower among physically active individuals in the total study population and particularly among those with i-IGT. The associations were attenuated and lost statistical significance after further
\end{abstract}

S. Engberg $(\bowtie) \cdot$ D. R. Witte $\cdot$ K. Borch-Johnsen

Steno Diabetes Center,

Niels Steensens vej 2,

2820 Gentofte, Denmark

e-mail: Segb@Steno.dk

C. Glümer · T. Jørgensen

Research Centre for Prevention and Health,

Glostrup, Denmark

T. Jørgensen

Faculty of Health Sciences, University of Copenhagen,

Copenhagen, Denmark

K. Borch-Johnsen

Faculty of Health Sciences, University of Aarhus,

Aarhus, Denmark adjustment for BMI. We observed no impact of physical activity on the progression to diabetes in individuals with i-IFG.

Conclusions/interpretation Physical activity was associated with a lower progression to diabetes in the total study population and in individuals with i-IGT, a condition primarily characterised by muscle insulin resistance. Physical activity did not predict progression to diabetes in individuals with i-IFG, a condition primarily characterised by hepatic insulin resistance. Our results suggest that there is a differential relationship between physical activity and progression to diabetes among those with i-IFG and i-IGT. Therefore, clinical trials testing the effect of physical activity on progression from i-IFG to diabetes are needed.

Trial registration: ClinicalTrials.gov ID No.: NCT00289237 Funding: The Danish Medical Research Council, the Danish Center for Evaluation and Health Technology Assessment, Novo Nordisk, Copenhagen County, the Danish Heart Foundation, the Danish Diabetes Association, the Danish Pharmaceutical Association, the Augustinus Foundation, the Ib Henriksen Foundation and the Becket Foundation.

Keywords Diabetes · Epidemiology · Impaired fasting glucose $\cdot$ Impaired glucose tolerance $\cdot$ Insulin resistance Physical activity · Population-based · Progression rate . Public health
Abbreviations
HOMA-IS HOMA of insulin sensitivity
IGT Impaired glucose tolerance
i-IFG Isolated impaired fasting glucose
i-IGT Isolated impaired glucose tolerance
IHD Ischaemic heart disease 
$\mathrm{ISI}_{0-120} \quad$ Insulin sensitivity index

NGT Normal glucose tolerance

\section{Introduction}

Intervention studies have demonstrated that lifestyle changes including increased physical activity can reduce the incidence of diabetes among individuals with impaired glucose tolerance (IGT) [1-3]. However, it is unknown whether these results can be extrapolated to individuals with isolated impaired fasting glucose (i-IFG) [4].

Prospective studies have shown that increased levels of physical activity can reduce progression to diabetes $[5,6]$. Recent population-based studies have examined the relationship between physical activity and overall progression to diabetes [7-9]. Most studies have classified physical activity into a two or three category variable not analysing dose-response relationships and not focusing on different types of physical activity (leisure, commuting and occupational) [10]. Both commuting and leisure-time physical activity are important from a public-health perspective [11], as both are modifiable and amenable to intervention. A separate consideration of physical activity types is necessary to quantify the potential impact of targeted interventions. Large population-based studies are needed to examine the relationship between physical activity and progression to diabetes and to study dose-response associations in different types of physical activity.

None of the mentioned population-based studies have analysed how physical activity affects progression from i-IFG or isolated IGT (i-IGT) to diabetes. Although i-IFG and i-IGT both represent intermediate stages of glucose intolerance and are insulin-resistant states with impaired beta cell function, they are distinct conditions with different pathophysiological aetiologies [12-15]. Clinical studies suggest that the site of insulin resistance varies between the two disorders. While individuals with i-IFG are characterised by hepatic insulin resistance with normal or near-normal muscle insulin sensitivity, individuals with i-IGT mainly have muscle insulin resistance with mild hepatic insulin resistance $[12,13,16]$.

Physical activity has been shown to be independently associated with lower $2 \mathrm{~h}$ plasma glucose but not fasting plasma glucose $[17,18]$, and in the Inter99 Study higher levels of physical activity predicted a decline in $2 \mathrm{~h}$ plasma glucose but not in fasting plasma glucose in men [19]. Physical activity can reduce muscle insulin resistance $[20,21]$; however, studies have also shown that physical activity is associated with a lower intrahepatic fat content [22], and that intrahepatic fat content is associated with hepatic insulin sensitivity [23, 24]. Consequently, a differ- ential effect of physical activity on progression to diabetes in individuals with i-IFG and i-IGT could be expected.

Therefore, the aim of our study was to analyse how strongly commuting and leisure-time physical activity affect progression to diabetes and to study whether this relationship is different in individuals with i-IFG and i-IGT.

\section{Methods}

Study design The Inter99 Study is a population-based primary prevention study on cardiovascular disease and type 2 diabetes. The study population comprised all 61,301 individuals born in 1939-40, 1944-45, 1949-50, 1954-55, 1959-60, 1964-65 and 1969-70 living in Copenhagen County, Denmark, on 2 December 1998. From the study population, an age- and sex-stratified random sample comprising 13,016 individuals was drawn. The sample was a priori randomised into two groups comprising $90 \%$ (high-intensity intervention) and 10\% (low-intensity intervention) [25].

Of the 13,016 individuals, 82 were not eligible because they had died or could not be traced. The remaining 12,934 were invited to a health-screening programme and a personal risk assessment of their absolute 10 year risk of developing ischaemic heart disease (IHD) by the Copenhagen Risk Score [25]. High-risk individuals were defined as individuals with an absolute risk of IHD in the upper quintile of their respective age and sex stratum or with one or more of the following risk factors: daily smoker, systolic $\mathrm{BP} \geq 160 \mathrm{mmHg} /$ antihypertensive therapy, total cholesterol $\geq 7.5 \mathrm{mmol} / \mathrm{l}, \quad \mathrm{BMI} \geq 30 \mathrm{~kg} / \mathrm{m}^{2}$, history of diabetes, or diabetes or IGT diagnosed at baseline. Based on the personal risk estimate, each individual was offered lifestyle counselling dealing with smoking, physical activity, diet and alcohol. High-risk individuals in the high-intensity intervention group were further offered lifestyle counselling in groups on smoking cessation or physical activity/diet with six meetings during a 4-6 month period, whereas high-risk individuals in the low-intensity intervention group were referred to usual care. Baseline data were collected from March 1999 until January 2001. The Inter99 Study and baseline results are described in detail elsewhere $[25,26]$.

All high-risk individuals were re-invited after 1 and 3 years for a health examination including a new risk assessment and lifestyle counselling. If still at high risk according to the risk assessment at the re-examination, individuals in the high-intensity intervention group were again offered lifestyle counselling in groups, and individuals in the low-intensity intervention group were again referred to usual care. All participants were re-invited for a final health examination 5 years after baseline [27]. 
Study procedure The participants filled in a questionnaire on health and lifestyle prior to the baseline examination. All participants were asked the following question on commuting physical activity: 'How much time do you spend walking, cycling or running on your way to and from work?': 'Less than 15 min'; ' $15-30$ min'; ' 30 min to $1 \mathrm{~h}$ '; ' $1 \mathrm{~h}$ or more'; or 'I do not work at the moment'. Individuals, who did not work, were excluded in the variable commuting physical activity. Leisuretime physical activity was assessed by the following question: 'In your leisure time, how many hours a week are you physically active including walks, bicycle rides and gardening, but not transportation to and from work?' with six answer categories ('none', 'approximately half an hour per week', 'approximately $1 \mathrm{~h}$ per week', 'approximately $2-3 \mathrm{~h}$ per week', 'approximately 4-6 h per week' and ' $7 \mathrm{~h}$ per week or more').

Total physical activity was calculated by summing responses to the question on commuting physical activity (converted into min/week using a 5 day working week) and leisure-time physical activity (converted into min/week). When the answer categories were intervals the middle value was used, and when the answer categories were open-ended the lowest value was used. The answer category 'I do not work at the moment' was assigned the value $0 \mathrm{~min}$ as regard to commuting physical activity in order not to exclude participants who did not work from the variable total physical activity. The variable total physical activity was grouped into four categories: 0-2 h/week, 2-4 h/week, 4-7 h/week, and $>7 \mathrm{~h} /$ week [11].

In the questionnaire, nationality was divided into Danish and other. A family history of diabetes was defined as having a first-degree relative with diabetes. Dietary intake was assessed by a validated food-frequency questionnaire of dietary intake during the previous month [28, 29].

Height was measured without shoes to the nearest $0.5 \mathrm{~cm}$, weight was measured with light clothing and without shoes to the nearest $0.1 \mathrm{~kg}$, and BMI was calculated as $\mathrm{kg} / \mathrm{m}^{2}[25,26]$.

After a minimum $8 \mathrm{~h}$ of fasting overnight, all participants without known diabetes underwent a standard OGTT (75 g anhydrous glucose in $250 \mathrm{ml}$ water) at each examination. Plasma glucose and serum insulin were measured in the fasting state and after $120 \mathrm{~min}$. Blood samples for glucose measurements were taken in heparinsodium fluoride tubes, immediately put on ice, centrifuged and plasma separated within $30 \mathrm{~min}$. Plasma glucose was analysed using the hexokinase/glucose-6-phosphate dehydrogenase method (Boehringer, Mannheim, Germany) $[25,26]$. Insulin was analysed with a fluoroimmunoassay technique (AutoDELFIA; Perkin Elmer-Wallace, Turku, Finland) [15].

All participants gave written informed consent before taking part in the study. The study was approved by the local ethics committee (KA 98 155) [25].
Study population and definitions A total of 6,906 individuals participated in the investigation, 122 individuals were excluded because of alcoholism, drug abuse or linguistic barriers, leaving 6,784 participants $(52.5 \%$ of those invited) for analysis at baseline $[25,26]$. In general, the participation rate was higher in younger women than in younger men, and it increased with increasing age until 55 years of age, after which it declined. The participation rates were identical in the high- and in the low-intensity intervention group [25].

Glucose tolerance status was classified according to the 1999 WHO criteria by a single OGTT [30], and IGT was divided into i-IGT and combined impaired fasting glucoseIGT. At baseline, 374 (5.5\%) were non-classifiable because of lack of either fasting plasma glucose or $2 \mathrm{~h}$ plasma glucose measurements, and $404(6.0 \%)$ had either selfreported diabetes or diabetes diagnosed by the OGTT [26], leaving 6,006 individuals without diabetes at baseline.

At the 5 year follow-up, 1,975 individuals were lost to follow-up $(n=1,926)$ or were non-classifiable $(n=49)$, leaving 4,031 individuals $(67.1 \%)$ with relevant data for the present analyses. To calculate the crude progression rates from baseline to 5 year follow-up in this study, the Inter99 Study was analysed as if it were a cohort study. At the 5 year examination, incident cases were defined as individuals with newly detected diabetes or known diabetes (self-reported or diagnosed at the 1 or 3 year examination) [27].

Insulin sensitivity was estimated by HOMA of insulin sensitivity (HOMA-IS), which is based on fasting values of glucose and insulin, and by the insulin sensitivity index (ISI ${ }_{0-120}$ ), which is based on fasting and $2 \mathrm{~h}$ values of glucose and insulin from the OGTT, as well as information on body weight $[15,31-33]$.

Statistical analyses Progression rates were estimated by dividing the number of incident cases by person-years at risk using interval-censoring [27]. We present rate ratios from Poisson regression analyses adjusted for relevant confounders. The physical activity group with the highest number of incident cases was used as the reference group in order to maximise the precision of the estimates. To account for non-linearity of the relationship between age and progression to diabetes, the continuous variables age and $\mathrm{age}^{2}$ were both entered into the models. In analyses relating physical activity to incidence of diabetes, a potential modifying effect of glucose class i-IFG vs i-IGT at baseline was tested. Exact $95 \%$ CIs were calculated for proportions [34]. The Wilcoxon rank sum test was used to compare group differences for continuous variables. $p<0.05$ was considered statistically significant. Statistical analyses were performed using SAS (Version 9.1; SAS Institute, Cary, NC, USA). 


\section{Results}

Individuals with follow-up (67.1\%) were more likely to be men, of Danish nationality, were older, had a higher daily energy intake, were more physically active during commuting and leisure time, had a lower BMI, were less often in the high-risk group, and were less often in the upper quintile of IHD risk compared with individuals lost to follow-up (all $p<0.01)$ (data not shown).

Almost two-thirds $(62.9 \%)$ of the participants spent $<15 \mathrm{~min} /$ day on commuting physical activity and $45 \%$ spent $<4 \mathrm{~h} /$ week on leisure-time physical activity. Individuals with i-IGT were less active during commuting or leisure time than individuals with i-IFG (Table 1).

Individuals with i-IFG had a lower level of HOMA-IS but a higher level of ISI $_{0-120}$ compared with individuals with i-IGT (both $p<0.001$ ) (Table 1). In individuals with normal glucose tolerance (NGT), the corresponding median values (interquartile range) of HOMA-IS $\left(1^{2} \mathrm{mmol}^{-1} \mathrm{pmol}^{-1}\right)$ and $\mathrm{ISI}_{0-120}\left(\mathrm{mg} \mathrm{l}^{2} \mathrm{mmol}^{-1} \mathrm{pmol}^{-1} \mathrm{~min}^{-1}\right)$ were $0.14(0.09$ $0.20)$ and $26.0(22.4-30.2)$, respectively. Individuals with NGT had higher levels of HOMA-IS and ISI $_{0-120}$ compared with individuals with either i-IFG or i-IGT (all $p<0.001$ ).

Total physical activity was associated with progression to diabetes in the total study population. Inactive individuals had more than twice (rate ratio $=2.28,95 \%$ CI 1.29 4.03) the risk of progression to diabetes compared with the most active when adjusted for age and sex. Further adjustment for nationality, family history of diabetes, intervention group, high- or low-risk group, and energy intake at baseline attenuated the association by $23 \%$ (rate ratio $=1.76,95 \%$ CI $0.98-3.14$ ) but there was still a doseresponse relationship between total physical activity and progression to diabetes (Fig. 1a).

Table 1 Baseline characteristics of the participants according to glucose tolerance status

\begin{tabular}{|c|c|c|c|}
\hline Variable & Total study population & i-IFG & i-IGT \\
\hline$n(\%$ of total $)$ & 4,031 & $359(8.9)$ & $354(8.8)$ \\
\hline $\operatorname{Men}(\%)^{\mathrm{a}}$ & 50.7 & 74.1 & 42.9 \\
\hline Age (years) & $46.4(7.6)$ & $49.2(6.8)$ & $48.6(7.6)$ \\
\hline Danish nationality $(\%)^{\mathrm{a}}$ & $96.0(95.4-96.6)$ & $97.5(95.3-98.8)$ & $92.0(88.6-94.6)$ \\
\hline Family history of diabetes (\%) & $17.0(15.8-18.2)$ & $18.7(14.8-23.1)$ & $20.6(16.5-25.2)$ \\
\hline Energy intake $(\mathrm{kJ} / \text { day })^{\mathrm{a}}$ & $9,866(3,488)$ & $10,219(3,532)$ & $9,776(4,211)$ \\
\hline \multicolumn{4}{|l|}{ Commuting physical activity (min/day) ${ }^{a, b}$} \\
\hline$<15(\%)$ & $62.9(61.3-64.5)$ & $63.7(58.1-68.9)$ & $69.5(63.9-74.7)$ \\
\hline $15-30(\%)$ & $22.3(21.0-23.7)$ & $18.3(14.3-23.0)$ & $20.7(16.2-25.8)$ \\
\hline $30-60(\%)$ & $10.0(9.0-11.0)$ & $11.2(8.0-15.1)$ & $7.1(4.5-10.7)$ \\
\hline$\geq 60(\%)$ & $4.7(4.1-5.5)$ & $6.8(4.3-10.2)$ & $2.7(1.2-5.3)$ \\
\hline \multicolumn{4}{|l|}{ Leisure-time physical activity (h/week) $)^{\mathrm{a}, \mathrm{c}}$} \\
\hline $0(\%)$ & $2.0(1.6-2.5)$ & $1.4(0.5-3.2)$ & $5.2(3.1-8.1)$ \\
\hline $0.5(\%)$ & $4.0(3.4-4.7)$ & $3.7(2.0-6.2)$ & $6.3(4.0-9.4)$ \\
\hline $1(\%)$ & $8.4(7.6-9.3)$ & $8.4(5.8-11.8)$ & $9.8(6.9-13.4)$ \\
\hline $2-3(\%)$ & $30.4(29.0-31.9)$ & $26.1(21.6-31.0)$ & $31.3(26.5-36.5)$ \\
\hline $4-6(\%)$ & $33.1(31.7-34.6)$ & $31.5(26.7-36.6)$ & $30.2(25.4-35.3)$ \\
\hline$\geq 7(\%)$ & $22.0(20.7-23.3)$ & $28.9(24.3-33.9)$ & $17.2(13.4-21.6)$ \\
\hline BMI $\left(\mathrm{kg} / \mathrm{m}^{2}\right)$ & $25.9(4.2)$ & $27.7(4.5)$ & $27.5(4.9)$ \\
\hline High-intensity intervention group (\%) & $89.1(88.1-90.1)$ & $85.8(81.7-89.2)$ & $88.7(84.9-91.8)$ \\
\hline High-risk group (\%) ${ }^{\mathrm{a}}$ & $49.8(48.3-51.4)$ & $57.1(51.8-62.3)$ & $100(99.0-100)$ \\
\hline Absolute risk of IHD in upper quintile of age and sex stratum (\%) & $10.8(9.8-11.8)$ & $12.8(9.5-16.7)$ & $15.8(12.2-20.0)$ \\
\hline HOMA-IS $\left(1^{2} \mathrm{mmol}^{-1} \mathrm{pmol}^{-1}\right)^{\mathrm{a}, \mathrm{d}}$ & $0.13[0.08-0.18]$ & $0.08[0.06-0.12]$ & $0.10[0.06-0.16]$ \\
\hline $\mathrm{ISI}_{0-120}\left(\mathrm{mg} \mathrm{l}^{2} \mathrm{mmol}^{-1} \mathrm{pmol}^{-1} \mathrm{~min}^{-1}\right)^{\mathrm{a}, \mathrm{e}}$ & $24.8[20.6-29.2]$ & $21.6[19.2-25.7]$ & $16.2[14.7-18.2]$ \\
\hline
\end{tabular}

Data are percentages $(95 \% \mathrm{CI})$, means (SD) or medians [interquartile range], unless otherwise indicated

${ }^{a}$ Statistically significant difference between i-IFG and i-IGT $(p<0.05)$

${ }^{\mathrm{b}} 429$ individuals were not working or had a missing value on commuting physical activity

${ }^{\mathrm{c}} 51$ individuals had a missing value on leisure-time physical activity

d 315 had a missing value on fasting insulin or had haemolysis in the blood sample

${ }^{\mathrm{e}} 604$ had missing values on fasting or $2 \mathrm{~h}$ insulin or had haemolysis in the blood sample 
a
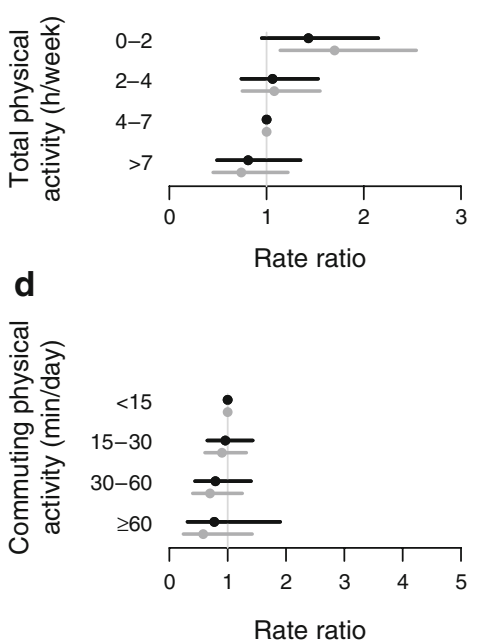

g

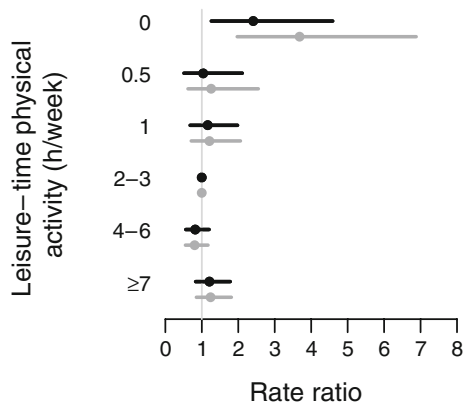

b
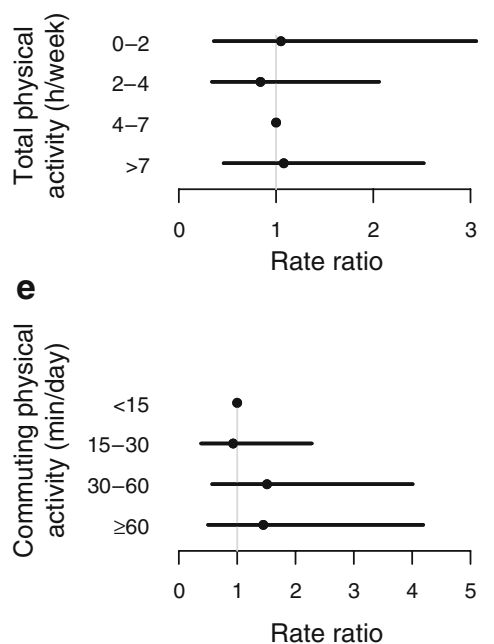

h

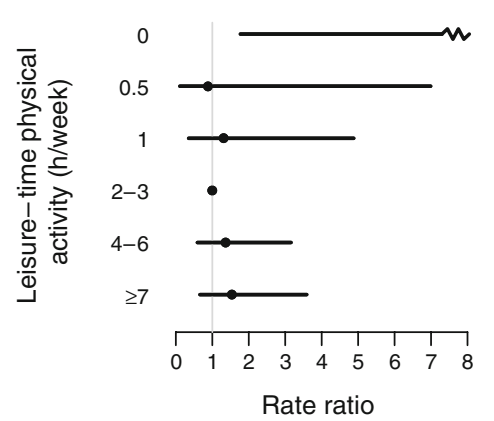

C
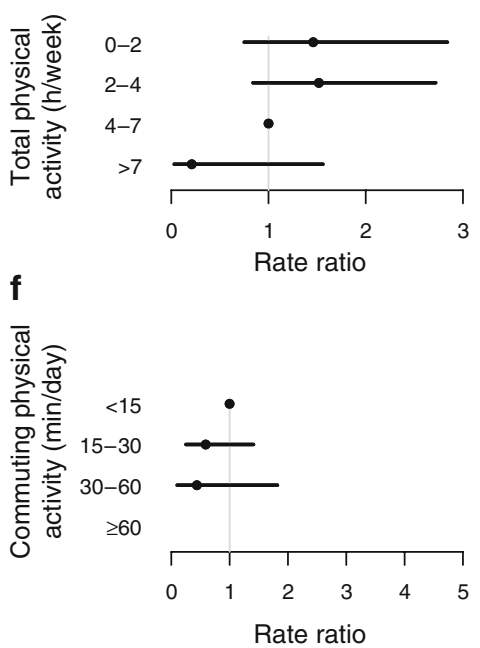

i

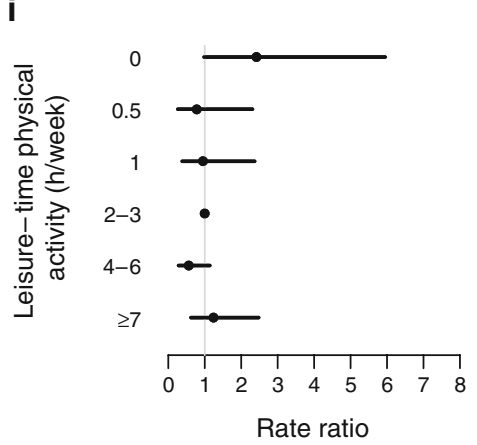

Fig. 1 Rate ratios for progression to diabetes according to level of physical activity in the total study population (a, d, $\mathbf{g}, n=189$ cases) and among individuals with i-IFG (b, e, h, $n=41$ cases) or i-IGT (c, f, i, $n=62$ cases). Of the 189 incident cases, 147 (87.8\%) had OGTTdiagnosed diabetes, the rest had self-reported diabetes. Grey circles, rate ratios adjusted for age and sex; black circles, adjusted for age and

When stratifying by glucose tolerance status and focusing on individuals with i-IFG or i-IGT, we found that those with i-IFG did not benefit from high levels of total physical activity, while inactive individuals with iIGT (0-2 h/week) had more than six times the risk of progression to diabetes compared with the most active with i-IGT (rate ratio=6.93, 95\% CI 0.91-53.02) (Fig. 1c). However, there was no significant difference between the trend tests in those with i-IFG or i-IGT ( $p$ for interaction= $0.09)$. In other words, although the $p$ value for trend in the i-IGT group is significant $(p=0.03)$, the trend is not significantly different from the trend in the i-IFG group (Fig. 1b,c).

Low levels of commuting physical activity were not significantly associated with progression to diabetes in the total study population or in individuals with i-IFG (Fig. 1d,e). In individuals with $\mathrm{i}-\mathrm{IGT}$, those with $>30 \mathrm{~min}$ of commuting physical activity per day had a 54\% lower risk of progression to diabetes compared with individuals who sex and for nationality, family history of diabetes, intervention group (high- or low-intensity), high- or low-risk group and energy intake at baseline. Range bars are 95\% CIs. $p$ values for trend are: a black $p=0.05$, grey $p=0.005 ; \mathbf{d}$ black $p=0.36$, grey $p=0.09 ;$ g black $p=0.23$, grey $p=0.05 ; \mathbf{b} p=0.81 ; \mathbf{e} p=0.37$; h $p=0.98$; c $p=0.03$; f $p=0.04$; i $p=0.26$

spent $<15 \mathrm{~min} /$ day in active commuting (rate ratio $=0.44$, 95\% CI 0.10-1.82) (Fig. 1f). The trend tests were significantly different among those with i-IFG and i-IGT ( $p$ for interaction $=0.046)$.

Leisure-time physical activity $(<7 \mathrm{~h}$ per week) was inversely associated with progression to diabetes in the total study population. Totally sedentary individuals during leisure time had a 4.6 times higher risk of progression to diabetes compared with individuals who were active (4-6 h/week) (rate ratio $=4.60,95 \%$ CI 2.45-8.64). The association was attenuated after further adjustment for nationality, family history of diabetes, intervention group, high- or low-risk group, and energy intake at baseline (rate ratio $=2.95,95 \%$ CI 1.53-5.68) but the dose-response relationship was unchanged (Fig. 1g). When stratified into i-IFG or i-IGT, the dose-response relationship between leisure-time physical activity ( $<7 \mathrm{~h} /$ week) and progression to diabetes disappeared, and there was no significant difference between the trend tests in the two groups ( $p$ for interaction=0.54). Totally 
sedentary individuals with i-IFG or i-IGT had an 8.49 (95\% CI 1.77-40.71) and a $2.42(95 \%$ CI $0.98-5.95)$ higher risk of progression to diabetes, respectively, compared with more active individuals $(2-3 \mathrm{~h} /$ week) (Fig. 1h,i).

When further adjusted for BMI, all the associations between total, commuting or leisure-time physical activity and progression to diabetes were attenuated. The associations between total or commuting physical activity and progression from i-IGT to diabetes both lost statistical significance ( $p$ for trend $=0.07$ and 0.06 , respectively). However, the dose-response pattern remained almost unchanged (data not shown).

\section{Discussion}

We found that high levels of total physical activity were inversely associated with the risk of progression to diabetes in the total study population and particularly in individuals with i-IGT. Total physical activity did not predict progression to diabetes in individuals with i-IFG. When we looked at commuting and leisure-time physical activity separately, commuting physical activity only showed a dose-response relationship with progression to diabetes in individuals with i-IGT. Leisure-time physical activity did not show a simple dose-response relationship with progression to diabetes. The deleterious relationship between being totally inactive during leisure time and progression to diabetes was pronounced for the total study population and for individuals with i-IFG. This may reflect the previously reported association between sedentary behaviour and an elevated risk of diabetes, independently of physical activity level $[35,36]$.

HOMA-IS, which is assessed by fasting values of glucose and insulin, is considered a measure of hepatic insulin sensitivity $[12,13,33]$. ISI ${ }_{0-120}$ is derived from plasma glucose and glucose concentrations during an OGTT and reflects both muscle and liver insulin sensitivity [13]. We have shown that individuals with i-IFG have a lower level of HOMA-IS but a higher level of ISI $_{0-120}$ compared with individuals with i-IGT. Furthermore, individuals with NGT have the highest levels of HOMA-IS and ISI $_{0-120}$. These findings suggest that individuals with i-IFG are characterised by predominantly hepatic insulin resistance compared with individuals with i-IGT, who have predominantly muscle insulin resistance. This is in accord with previous studies $[12-14,16]$.

Commuting and leisure-time physical activity are inversely associated with BMI in the Inter99 Study [11], and prospective studies have shown that physical activity can reduce the risk of diabetes independently of BMI or other measures of overweight/obesity $[5,6,37-40]$. However, physical activity may also reduce total body fat and/or its distribution and facilitate weight maintenance, which indirectly reduces the risk of diabetes [35, 40-42]. Therefore, statistical adjustment for BMI or other measures of overweight/obesity attenuates the association between physical activity and development of diabetes in most studies [35, 38, 40, 43] and in our study. That is, overweight/obesity can be considered both a potential confounder and a potential mediator in the relationship between physical activity and progression to diabetes.

Most population-based studies have only examined overall progression to diabetes and have therefore not had the opportunity to study a possible differential relationship of physical activity in individuals with i-IFG or i-IGT. Furthermore, most of the studies have assessed overall physical activity. From a public-health point of view, it makes sense to divide overall physical activity into relevant types (leisure-time, commuting and occupational).

In the large Australian AusDiab Study, overall physical inactivity was associated with incident diabetes in multivariate analyses including adjustment for waist circumference as a measure of obesity [8]. In the Italian Bruneck Study, however, physical activity (high vs low levels) was not significantly related to diabetes incidence in univariate analyses [7], and in the Spanish Asturias Study, low physical activity failed to predict progression to diabetes in a multivariate model which included adjustment for BMI [9].

Strengths of this population-based follow-up study include the large number of participants with 5 year follow-up data $(n=4,031)$, assessment of glucose tolerance status by an OGTT at baseline and at 5 year follow-up, availability of measures of insulin sensitivity (HOMA-IS and $\mathrm{ISI}_{0-120}$ ) and the separate analysis of progression from $\mathrm{i}$-IFG or i-IGT to diabetes. A further strength is the detailed information on commuting and leisure-time physical activity in the questionnaire, which gives us an opportunity to observe a dose-response relationship. We have not studied the relationship between occupational physical activity and progression to diabetes because there is no information on total duration/time spent at occupational physical activity in the Inter99 Study.

The study-participation rate $(52.5 \%)$ was relatively low and it is lower than or comparable to similar studies $(63.6 \%$ in Asturias [9] and 55\% in AusDiab [8]). A total of 67.1\% of the participants were followed up and had a complete glucose tolerance status assessment at 5 year follow-up, which is comparable to other studies $[8,9]$. The participation rate and the loss to follow-up are unlikely to have affected the internal validity of our findings, but may affect the generalisability of the results.

In accordance with the WHO 1999 criteria, the classification of glucose tolerance status in this epidemiological study was based on a single OGTT examination [30]. 
Because of the known high intra-individual variation in plasma glucose levels, especially for $2 \mathrm{~h}$ plasma glucose, it is likely that some individuals may have been misclassified owing to normal day-to-day variation in plasma glucose $[15,44]$. This random misclassification can underestimate the true relationship between physical activity and progression to diabetes. Furthermore, it is impossible to know whether participants were truly fasting for at least $8 \mathrm{~h}$ prior to the blood sampling. If the fasting period was $<8 \mathrm{~h}$, individuals with NGT may be misclassified as i-IFG, which can underestimate the true association between physical activity and progression from i-IFG to diabetes.

We analysed the Inter99 Study as if it were a cohort study, although it is designed as an intervention study. The information on physical activity and other baseline characteristics was collected before the participants were allocated to the high- or the low-risk group and before the lifestyle intervention began. Therefore, the association between these characteristics and progression to diabetes cannot be biased by the intervention. The intervention could affect the progression rates differentially among individuals with i-IFG and i-IGT because the diagnosis IGT was one of the criteria for being at high risk. We performed a sensitivity analysis restricted to the high-risk segment of the individuals with i-IFG $(57.1 \%)$ and the trend tests remained statistically non-significant (data not shown). In the calculation of the rate ratios, we adjusted for the intervention. Furthermore, we did not observe a difference between the progression rates in individuals in the low- compared with the high-intensity intervention group (rate ratio $=0.92$, 95\% CI 0.58-1.44).

Our physical activity questionnaire has not been validated against objective measures of physical activity (e.g. the double-labelled water method or direct or indirect calorimetry). However, these objective measures are expensive and cumbersome methods that cannot validate physical activity over longer periods [10]. The questionnaire has been shown to be associated with HDLcholesterol and inversely associated with LDL-cholesterol, triacylglycerol, waist circumference and BMI in the Inter99 Study [11]. Furthermore, our aim was to rank the study population according to level of physical activity and not to study their absolute level of physical activity.

Physical activity is difficult to assess in a reliable and valid way in large population-based studies. Questionnaires are often the only feasible method, although they are associated with bias and thereby inaccurate assessment of physical activity, which is likely to attenuate the associations studied [10]. Recalling physical activity is a highly complex cognitive task; social desirability may lead to over-reporting of physical activity [45], and simple questionnaires are often more reliable and valid than more complex ones [10]. Furthermore, commuting and leisure- time physical activity are affected by seasonal variation in Denmark. As our questionnaire asked for habitual physical activity level, we have not included seasonality in our analysis. Commuting physical activity is characterised by regularity during the working week and the amount of time spent is relatively easy to recall. Therefore, we believe that our assessment of commuting physical activity is quite unbiased. Leisure-time physical activity is more difficult to recall because of its unstructured and complex nature. However, individuals who are totally inactive in their leisure time or during commuting may find it easier to report inactivity compared with individuals who have a varying physical activity pattern. The imprecise assessment of leisure-time physical activity ( $>0 \mathrm{~h} / \mathrm{week})$ is likely to attenuate the associations and can explain why we did not observe a relationship between increasing levels of leisuretime physical activity and progression from i-IGT to diabetes. It has previously been shown that higher levels of leisure-time physical activity as well as commuting and occupational physical activity were associated with a lower risk of diabetes [43].

We believe that commuting and leisure-time physical activity affect the progression rates through the same physiological mechanisms. Nevertheless, from a publichealth perspective it is relevant to divide physical activity into different types [43]. Benefits of different physical activities depend not only on the specific activity but also on the activity it displaces [46]. Lack of time is an important barrier for being physically active [47]. The Inter99 population comprised 30- to 60-year-old individuals, many with a busy working life and little spare time, and almost two-thirds $(62.9 \%)$ of the participants spent $<15 \mathrm{~min} /$ day on commuting physical activity or did not work. Therefore, some individuals might prefer activities that can be incorporated into daily living activities. Physical activity in transportation to and from work could be such an activity for some individuals, as some might find it less time-consuming and more convenient to be physically active during commuting than at leisure time [11]. We have shown that increased commuting and leisure-time physical activity in the population is likely to have a positive impact on the prevention of diabetes. Our findings indicate that public-health strategies aiming to reduce progression to diabetes by increasing physical activity may benefit from focusing on commuting physical activity besides leisuretime physical activity [11].

Intervention studies have demonstrated that lifestyle changes including increased physical activity can reduce the incidence of diabetes among individuals with IGT [1-3]. However, no trial evidence has been published on the effect of physical activity on progression from i-IFG to diabetes. Although we cannot exclude the possibility that the lack of a relationship between physical activity and 
progression to diabetes in the i-IFG group may be attributable to limited power, our findings suggest that there is a differential relationship between physical activity and progression to diabetes among those with i-IFG or i-IGT. Therefore, clinical trials testing the effect of physical activity on progression from i-IFG to diabetes are needed.

Acknowledgements We would like to thank all the participants in the survey, as well as the staff from the Research Centre for Prevention and Health and from the laboratory at Steno Diabetes Center for their dedicated effort in making the Inter99 Study possible. The study was initiated by T. Jørgensen (Principal Investigator [PI]), K. Borch-Johnsen (co-PI), T. Thomsen and H. Ibsen. The present steering committee comprises the two former and C. Pisinger. This study was supported by grants from the Danish Medical Research Council, the Danish Center for Evaluation and Health Technology Assessment, Novo Nordisk, Copenhagen County, the Danish Heart Foundation, the Danish Diabetes Association, the Danish Pharmaceutical Association, the Augustinus Foundation, the Ib Henriksen Foundation and the Becket Foundation.

Duality of interest K. Borch-Johnsen is head of the Steno Diabetes Center, a hospital integrated into the Danish National Health Care Service, but owned by Novo Nordisk. K. Borch-Johnsen holds shares in Novo Nordisk. S. Engberg, C. Glümer and D. R. Witte hold individual shares in Novo Nordisk. T. Jørgensen declares there is no duality of interest associated with this manuscript.

\section{References}

1. Pan XR, Li GW, Hu YH et al (1997) Effects of diet and exercise in preventing NIDDM in people with impaired glucose tolerance. The Da Qing IGT and Diabetes Study. Diabetes Care 20:537-544

2. Tuomilehto J, Lindström J, Eriksson JG et al (2001) Prevention of type 2 diabetes mellitus by changes in lifestyle among subjects with impaired glucose tolerance. N Engl J Med 344:1343-1350

3. Diabetes Prevention Program Research Group (2002) Reduction in the incidence of type 2 diabetes with lifestyle intervention or metformin. N Engl J Med 346:393-403

4. Yates T, Khunti K, Bull F, Gorely T, Davies MJ (2007) The role of physical activity in the management of impaired glucose tolerance: a systematic review. Diabetologia 50:1116-1126

5. Hu G, Lakka TA, Kilpeläinen TO, Tuomilehto J (2007) Epidemiological studies of exercise in diabetes prevention. Appl Physiol Nutr Metab 32:583-595

6. Jeon CY, Lokken RP, Hu FB, van-Dam RM (2007) Physical activity of moderate intensity and risk of type 2 diabetes: a systematic review. Diabetes Care 30:744-752

7. Bonora E, Kiechl S, Willeit J et al (2004) Population-based incidence rates and risk factors for type 2 diabetes in white individuals: the Bruneck study. Diabetes 53:1782-1789

8. Magliano DJ, Barr ELM, Zimmet PZ et al (2008) Glucose indices, health behaviors, and incidence of diabetes in Australia: The Australian Diabetes, Obesity and Lifestyle Study. Diabetes Care 31:267-272

9. Valdés S, Botas P, Delgado E, Alvarez F, Cadórniga FD (2007) Population-based incidence of type 2 diabetes in northern Spain: the Asturias Study. Diabetes Care 30:2258-2263

10. Shephard RJ (2003) Limits to the measurement of habitual physical activity by questionnaires. Br J Sports Med 37:197-206

11. von Huth Smith L, Borch-Johnsen K, Jørgensen T (2007) Commuting physical activity is favourably associated with biological risk factors for cardiovascular disease. Eur J Epidemiol 22:771-779

12. Abdul-Ghani MA, Jenkinson CP, Richardson DK, Tripathy D, DeFronzo RA (2006) Insulin secretion and action in subjects with impaired fasting glucose and impaired glucose tolerance. Diabetes 55:1430-1435

13. Abdul-Ghani MA, Tripathy D, DeFronzo RA (2006) Contributions of beta-cell dysfunction and insulin resistance to the pathogenesis of impaired glucose tolerance and impaired fasting glucose. Diabetes Care 29:1130-1139

14. Hanefeld M, Koehler C, Fuecker K, Henkel E, Schaper F, Temelkova-Kurktschiev T (2003) Insulin secretion and insulin sensitivity pattern is different in isolated impaired glucose tolerance and impaired fasting glucose: The Risk Factor in Impaired Glucose Tolerance for Atherosclerosis and Diabetes Study. Diabetes Care 26:868-874

15. Færch K, Vaag A, Holst JJ, Hansen T, Jørgensen T, BorchJohnsen K (2009) Natural history of insulin sensitivity and insulin secretion in the progression from normal glucose tolerance to impaired fasting glycemia and impaired glucose tolerance: The Inter99 Study. Diabetes Care 32:439-444

16. Weyer C, Bogardus C, Pratley RE (1999) Metabolic characteristics of individuals with impaired fasting glucose and/or impaired glucose tolerance. Diabetes 48:2197-2203

17. Healy GN, Dunstan DW, Shaw JE, Zimmet PZ, Owen N (2006) Beneficial associations of physical activity with 2-h but not fasting blood glucose in Australian adults: The AusDiab Study. Diabetes Care 29:2598-2604

18. Kriska AM, LaPorte RE, Pettitt DJ et al (1993) The association of physical activity with obesity, fat distribution and glucose intolerance in Pima Indians. Diabetologia 36:863-869

19. Færch K, Vaag A, Witte DR, Jørgensen T, Pedersen O, BorchJohnsen K (2009) Predictors of future fasting and 2-h post-OGTT plasma glucose levels in middle-aged men and women-the Inter99 study. Diabet Med 26:377-383

20. Houmard JA, Tanner CJ, Slentz CA, Duscha BD, McCartney JS, Kraus WE (2004) Effect of the volume and intensity of exercise training on insulin sensitivity. J Appl Physiol 96:101-106

21. Perseghin G, Price TB, Petersen KF et al (1996) Increased glucose transport-phosphorylation and muscle glycogen synthesis after exercise training in insulin-resistant subjects. N Engl J Med 335:1357-1362

22. Luzi L (2007) Habitual physical activity is associated with intrahepatic fat content in humans. Diabetes Care 30:683-688

23. Seppala-Lindroos A, Vehkavaara S, Hakkinen AM et al (2002) Fat accumulation in the liver is associated with defects in insulin suppression of glucose production and serum free fatty acids independent of obesity in normal men. J Clin Endocrinol Metab 87:3023-3028

24. Marchesini G, Brizi M, Bianchi G et al (2001) Nonalcoholic fatty liver disease. Diabetes 50:1844-1850

25. Jørgensen T, Borch-Johnsen K, Thomsen TF, Ibsen H, Glümer C, Pisinger C (2003) A randomized non-pharmacological intervention study for prevention of ischaemic heart disease: baseline results Inter99. Eur J Cardiovasc Prev Rehabil 10:377-386

26. Glümer C, Jørgensen T, Borch-Johnsen K (2003) Prevalences of diabetes and impaired glucose regulation in a Danish population: The Inter99 study. Diabetes Care 26:2335-2340

27. Engberg S, Vistisen D, Lau C et al (2009) Progression to impaired glucose regulation and diabetes in the population-based Inter99 Study. Diabetes Care 32:606-611

28. Lau C, Færch K, Glümer C et al (2005) Dietary glycemic index, glycemic load, fiber, simple sugars, and insulin resistance: the Inter99 study. Diabetes Care 28:1397-1403

29. Toft U, Kristoffersen L, Ladelund S et al (2008) Relative validity of a food frequency questionnaire used in the Inter99 study. Eur J Clin Nutr 62:1038-1046 
30. World Health Organization (1999) Definition, diagnosis and classification of diabetes mellitus and its complications. Report of a WHO consultation, Part 1: Diagnosis and classification of diabetes mellitus. World Health Organization, Geneva

31. Matthews DR, Hosker JP, Rudenski AS, Naylor BA, Treacher DF, Turner RC (1985) Homeostasis model assessment: insulin resistance and beta-cell function from fasting plasma glucose and insulin concentrations in man. Diabetologia 28:412-419

32. Gutt M, Davis CL, Spitzer SB et al (2000) Validation of the insulin sensitivity index $\left(\mathrm{IS}_{\mathrm{I} 0}, 120\right)$ : comparison with other measures. Diabetes Res Clin Pract 47:177-184

33. Radikova Z (2003) Assessment of insulin sensitivity/resistance in epidemiological studies. Endocr Regul 37:189-194

34. Harte D (2002) Non asymptotic binomial confidence intervals. http://www.statsresearch.co.nz/pdf/confint.pdf, accessed 24 June 2009

35. Hu FB, Leitzmann MF, Stampfer MJ, Colditz GA, Willett WC, Rimm EB (2001) Physical activity and television watching in relation to risk for type 2 diabetes mellitus in men. Arch Intern Med 161:1542-1548

36. Hu FB, Li TY, Colditz GA et al (2003) Television watching and other sedentary behaviors in relation to risk of obesity and type 2 diabetes mellitus in women. JAMA 289:1785-1791

37. Helmrich SP, Ragland DR, Leung RW, Paffenbarger RS (1991) Physical activity and reduced occurrence of non-insulin-dependent diabetes mellitus. N Engl J Med 325:147

38. Kriska AM, Saremi A, Hanson RL et al (2003) Physical activity, obesity, and the incidence of type 2 diabetes in a high-risk population. Am J Epidemiol 158:669-675
39. Manson JE, Rimm EB, Stampfer MJ et al (1991) Physical activity and incidence of non-insulin-dependent diabetes mellitus in women. Lancet 338:774

40. Hu FB, Sigal RJ, Rich-Edwards JW et al (1999) Walking compared with vigorous physical activity and risk of type 2 diabetes in women: a prospective study. JAMA 282:1433-1439

41. Gill JM, Cooper AR (2008) Physical activity and prevention of type 2 diabetes mellitus. Sports Med 38:807-824

42. Hamman RF, Wing RR, Edelstein SL et al (2006) Effect of weight loss with lifestyle intervention on risk of diabetes. Diabetes Care 29:2102-2107

43. Hu G, Qiao Q, Silventoinen K et al (2003) Occupational, commuting, and leisure-time physical activity in relation to risk for type 2 diabetes in middle-aged Finnish men and women. Diabetologia 46:322-329

44. Mooy JM, Grootenhuis PA, de Vries H et al (1996) Intraindividual variation of glucose, specific insulin and proinsulin concentrations measured by two oral glucose tolerance tests in a general Caucasian population: the Hoorn Study. Diabetologia 39:298-305

45. Sallis JF, Saelens BE (2000) Assessment of physical activity by self-report: status, limitations, and future directions. Res Q Exerc Sport 71:1-14

46. Mekary RA, Willett WC, Hu FB, Ding EL (2009) Isotemporal substitution paradigm for physical activity epidemiology and weight change. Am J Epidemiol 170:519-527

47. Lian WM, Gan GL, Pin CH, Wee S, Ye HC (1999) Correlates of leisure-time physical activity in an elderly population in Singapore. Am J Public Health 89:1578-1580 